\title{
TNFSF4 Gene Variations Are Related to Early-Onset Autoimmune Thyroid Diseases and Hypothyroidism of Hashimoto's Thyroiditis
}

\author{
Rong-Hua Song ${ }^{1,+}$, Qiong Wang ${ }^{2,+}$, Qiu-Ming Yao ${ }^{1}$, Xiao-Qing Shao ${ }^{1}$, Ling Li ${ }^{1}$, Wen Wang ${ }^{1}$, \\ Xiao-Fei An ${ }^{1}$, Qian Li ${ }^{1}$ and Jin-An Zhang ${ }^{1, *}$ \\ 1 Department of Endocrinology, Jinshan Hospital of Fudan University, No. 1508 Longhang Road, Jinshan \\ District, Shanghai 201508, China; someonesrh66@163.com (R.-H.S.); 14211270001@fudan.edu.cn (Q.-M.Y.); \\ shaoxq2015@163.com (X.-Q.S.); alana3344@126.com (L.L.); wwen910108@163.com (W.W.); \\ anxiaofei2000@163.com (X.-F.A.); ellen38668@163.com (Q.L.) \\ 2 The hemodialysis center of Nephropathy Department, Shaanxi Provincial People's Hospital, \\ No. 256 West Youyi Road, Beilin District, Xi'an 710068, China; wq9930@126.com \\ * Correspondence: zhangjinan@hotmail.com; Tel.: +86-21-5703-9815; Fax: +86-21-6722-6910 \\ + These authors contributed equally to this work.
}

Academic Editor: Daniela Gabriele Grimm

Received: 10 July 2016; Accepted: 16 August 2016; Published: 20 August 2016

\begin{abstract}
The aim of the current study was to examine whether the polymorphism loci of the tumor necrosis factor superfamily member 4 (TNFSF4) gene increase the risk of susceptibility to autoimmune thyroid diseases (AITDs) in the Han Chinese population, and a case-control study was performed in a set of 1,048 AITDs patients and 909 normal healthy controls in the study. A total of four tagging single nucleotide polymorphisms (SNPs) in the TNFSF4 region, including rs7514229, rs1234313, rs16845607 and rs3850641, were genotyped using the method of ligase detection reaction. An association between GG genotype of rs3850641 in TNFSF4 gene and AITDs was found $(p=0.046)$. Additionally, the clinical sub-phenotype analysis revealed a significant association between GG genotype in rs7514229 and AITDs patients who were $\leq 18$ years of age. Furthermore, rs 3850641 variant allele $G$ was in strong association with hypothyroidism in Hashimoto's thyroiditis (HT) $(p=0.018)$. The polymorphisms of the TNFSF4 gene may contribute to the susceptibility to AITDs pathogenesis.
\end{abstract}

Keywords: tumor necrosis factor superfamily member 4 (TNFSF4); single nucleotide polymorphism (SNP); autoimmune thyroid diseases (AITDs); Graves' disease (GD); Hashimoto's thyroiditis (HT)

\section{Introduction}

Autoimmune thyroid diseases (AITDs) are a group of organ-specific and polygenic inherited autoimmune diseases, with an estimated prevalence of up to $1 \%-5 \%$ of the general population [1]. AITDs mainly consist of two clinical subtypes of Graves' disease (GD) and Hashimoto's thyroiditis (HT). GD is predominantly characterized by a variable combination of hyperthyroidism, diffused goiter and high level of thyroid stimulating hormone receptor antibody (TRAb). Meanwhile, some GD patients may present extrathyroidal manifestations, including ophthalmopathy, pretibial myxedema and clubbed fingers. Clinical features of HT include the presence of antibody against thyroid peroxidase (TPOAb) or thyroglobulin (TgAb). Additionally, some patients with HT harbor extensive apoptosis of thyrocytes leading to hypothyroidism. Although there are some common characteristics in GD and HT, such as destruction of thyroid tissue and the existence of circulating thyroid autoantibodies including $\mathrm{TRAb}, \mathrm{TPOAb}$ and $\mathrm{TgAb}$, the clinical presentations and mechanisms of the two subtypes are different from each other to some extent; for example, our previous studies found that GD, HT or even Graves' ophthalmopathy (GO) have specific genetic backgrounds [2,3]. The pathogenesis of AITDs remains 
unclear, although there is much evidence demonstrating that the interaction between genetic factors and environmental components may be involved in their etiology $[4,5]$.

More recently, an increasing body of research has confirmed that several specific genes are associated with multiple autoimmune diseases [6,7], implicating that many autoimmune diseases may share some genetic risk factors. For instance, TNFAIP3 has been identified to be related to the genetic etiology of systemic lupus erythematosus (SLE) [8], rheumatoid arthritis (RA) [9], systemic sclerosis (SSc) [10]. Additionally, we also found the relationship between this gene and GD [11]. All these data documented that variants in several genes probably contribute to dysregulation of common immune pathways, and then are involved in the pathological procedure of diverse autoimmune diseases.

The tumor necrosis factor superfamily member 4 (TNFSF4) gene encodes a cytokine (OX40L), which is expressed on antigen-presenting cells (APCs) to provide co-stimulatory signals to T cells. In recent years, the TNFSF4 gene polymorphisms have been reported to be an important predisposition factor to SLE [12], RA [6], SSc [13] and primary Sjogren's syndrome (pSS) [14]. However, to date, whether TNFSF4 gene variations are associated with AITDs has not been investigated.

In the present study, we evaluated whether mutations in TNFSF4 gene are genetically predisposed in Han Chinese populations to AITDs via a case-control study. Single nucleotide polymorphisms (SNPs) tagging four independent susceptibility loci were genotyped in a large cohort of AITDs patients and normal healthy controls. We also analyzed the association between each polymorphism locus and the predisposition to different subtypes of AITDs, including GD, HT and ophthalmopathy.

\section{Results}

\subsection{Clinical Phenotype Analysis}

The clinical characteristics of the AITDs cohort are displayed in Table 1. Among the investigated 1,048 AITDs patients, 693 were GD patients including $30.736 \%$ male and $69.264 \%$ female, with mean disease-onset age of $34.010 \pm 14.395$ years old; 355 were HT patients, including $12.676 \%$ male and $87.324 \%$ female, with mean disease-onset age of $32.720 \pm 13.511$ years old. There were 162 $(15.458 \%)$ teenaged AITDs patients with disease-onset age $\leq 18$ years old, $130(12.405 \%)$ AITDs patients with ophthalmopathy, 198 (55.775\%) HT patients with hypothyroidism, and 216 (20.611\%) AITDs patients, comprised of $143 \mathrm{GD}$ patients and 73 HT patients, with family history.

Table 1. Clinical data of AITDs patients and controls.

\begin{tabular}{ccccc}
\hline Clinical Phenotype & AITDs (\%) & GD (\%) & HT (\%) & Control (\%) \\
\hline Number & 1048 & 693 & 355 & 909 \\
\hline Gender & - & - & - & - \\
Male & $260(24.809)$ & $213(30.736)$ & $45(12.676)$ & $314(34.543)$ \\
Female & $788(75.191)$ & $480(69.264)$ & $310(87.324)$ & $595(65.457)$ \\
Onset of age & $33.570 \pm 14.108$ & $34.010 \pm 14.395$ & $32.720 \pm 13.511$ & - \\
$\leq 18$ years & $162(15.458)$ & $113(16.306)$ & $49(13.803)$ & - \\
$\geq 19$ years & $886(84.542)$ & $580(83.694)$ & $306(86.197)$ & - \\
\hline Ophthalmopathy & - & - & - & - \\
$(+)$ & $130(12.405)$ & $124(17.893)$ & $6(1.690)$ & - \\
$(-)$ & $918(87.595)$ & $569(82.107)$ & $349(98.310)$ & - \\
\hline Family history & - & - & - & - \\
$(+)$ & $216(20.611)$ & $143(20.635)$ & $73(20.563)$ & - \\
$(-)$ & $832(79.389)$ & $550(79.365)$ & $282(79.437)$ & - \\
\hline
\end{tabular}

\subsection{Allelic and Genotypic Association}

There is a Hardy-Weinberg equilibrium (HWE) in the genotype distributions of TNFSF4 SNPs in the control group $(p>0.05)$. Additionally, we evaluated the HWE for the loci in our AITDs cases; 
the HWE of the four loci for AITDs cases all had $p$-value higher than 0.01. In addition, variant genotype GG of rs3850641 in TNFSF4 gene is associated with AITDs $(p=0.046)$, as shown in Table 2. Further analysis found that the frequencies of TT genotype in rs7514229 and GG genotype in rs3850641 were lower in the AITDs group than the control group (Table 3), which suggested that people with these genotypes are less susceptible to AITDs $(p=0.016, O R=0.236,95 \% \mathrm{CI}=0.066-0.850$ and $p=0.027$, $O R=0.492,95 \% C I=0.259-0.935$, respectively). Those subjects whose genotypes of the four loci failed to be determined were ruled out from the statistical analysis. Moreover, the frequency of genotype GG in rs3850641 was lower in GD patients than the control group in analysis of sub-clinical types of AITDs (GD and HT) although without statistical significance $(p=0.086)$, as shown in Table 4.

Table 2. Allele and genotype distribution of TNFSF4 in AITDs patients and controls.

\begin{tabular}{ccccc}
\hline SNP ID & Control & AITDs & $p$ & OR (95\% CI) \\
\hline rs7514229 & - & - & - & - \\
GG & $732(81.424)$ & $838(81.518)$ & 0.052 & - \\
TT & $11(1.224)$ & $3(0.292)$ & - & - \\
GT & $156(17.353)$ & $187(18.191)$ & - & $1.061(0.856-1.314)$ \\
G & $1620(90.100)$ & $1863(90.613)$ & 0.590 & - \\
T & $178(9.900)$ & $193(9.387)$ & - & - \\
rs1234313 & - & - & - & - \\
AA & $365(40.466)$ & $445(42.871)$ & 0.536 & - \\
GG & $110(12.195)$ & $117(11.272)$ & - & - \\
AG & $427(47.339)$ & $476(45.857)$ & - & - \\
A & $1157(64.135)$ & $1366(65.800)$ & 0.278 & - \\
G & $647(35.865)$ & $710(34.200)$ & - & - \\
rs16845607 & - & - & - & - \\
AA & $4(0.443)$ & $5(0.480)$ & 0.993 & - \\
GG & $797(88.359)$ & $920(88.292)$ & - & - \\
AG & $101(11.197)$ & $117(11.228)$ & - & - \\
$A$ & $109(6.042)$ & $127(6.094)$ & 0.946 & - \\
G & $1695(93.958)$ & $1957(93.906)$ & - & - \\
rs3850641 & - & - & - & - \\
GG & $26(2.879)$ & $15(1.438)$ & 0.046 & - \\
$A A$ & $660(73.090)$ & $750(71.908)$ & - & - \\
$A G$ & $217(24.031)$ & $278(26.654)$ & - & - \\
G & $269(14.895)$ & $308(14.765)$ & 0.910 & $0.990(0.829-1.182)$ \\
$A$ & $1537(85.105)$ & $1778(85.235)$ & - & - \\
\hline & & & & - \\
\hline
\end{tabular}

Table 3. Genotype frequency of TNFSF4 loci in AITDs patients and controls.

\begin{tabular}{ccccccc}
\hline SNP Name & Genotype & Control (\%) & AITDs (\%) & $p$ & OR & 95\% CI \\
\hline & GG & $732(81.424)$ & $838(81.518)$ & \multirow{2}{*}{0.958} & 1.006 & $0.799-1.267$ \\
& TT + GT & $167(18.576)$ & $190(18.482)$ & & & \\
rs7514229 & TT & $11(1.233)$ & $3(0.292)$ & 0.016 & 0.236 & $0.066-0.850$ \\
& GG + GT & $888(98.776)$ & $1025(99.708)$ & & & \\
& GT & $156(17.353)$ & $187(18.191)$ & 0.631 & 1.059 & $0.838-1.339$ \\
& GG + TT & $743(82.647)$ & $841(81.809)$ & & & \\
\hline & AA & $365(40.466)$ & $445(42.871)$ & \multirow{2}{*}{0.284} & 1.104 & $0.921-1.323$ \\
& GG + AG & $537(59.534)$ & $593(57.129)$ & & & \\
rs1234313 & GG & $110(12.195)$ & $117(11.272)$ & 0.528 & 0.915 & $0.693-1.206$ \\
& AA + AG & $792(87.805)$ & $921(88.728)$ & & & \\
& AG & $427(47.339)$ & $476(45.857)$ & 0.514 & 0.943 & $0.788-1.126$ \\
\hline
\end{tabular}


Table 3. Cont

\begin{tabular}{ccccccc}
\hline SNP Name & Genotype & Control (\%) & AITDs (\%) & $p$ & OR & 95\% CI \\
\hline & AA & $4(0.443)$ & $5(0.480)$ & \multirow{2}{*}{0.906} & 1.082 & $0.290-4.049$ \\
& GG+AG & $898(99.557)$ & $1037(99.520)$ & & & \\
rs16845607 & GG & $797(88.359)$ & $920(88.292)$ & 0.963 & 0.993 & $0.752-1.311$ \\
& AA + AG & $105(11.641)$ & $122(11.708)$ & & & \\
& AG & $101(11.197)$ & $117(11.228)$ & 0.983 & 1.003 & $0.756-1.330$ \\
& AA + GG & $801(88.803)$ & $925(88.772)$ & & & \\
\hline & GG & $26(2.879)$ & $15(1.438)$ & \multirow{2}{*}{0.027} & 0.492 & $0.259-0.935$ \\
rs3850641 & AA + AG & $877(97.121)$ & $1028(98.562)$ & & & \\
& AA & $660(73.090)$ & $750(71.908)$ & 0.561 & 0.943 & $0.772-1.151$ \\
& GG + AG & $243(26.910)$ & $293(28.092)$ & & & \\
& AG & $217(24.031)$ & $278(26.654)$ & 0.185 & 1.149 & $0.935-1.410$ \\
\hline
\end{tabular}

Table 4. Distribution of genotype and allele of TNFSF4 gene in sub-clinical types of AITDs patients and controls.

\begin{tabular}{|c|c|c|c|c|c|c|c|}
\hline SNP & Control & GD & $p$ & OR $(95 \% \mathrm{CI})$ & HT & $p$ & $O R(95 \% \mathrm{CI})$ \\
\hline rs7514229 & - & - & - & - & - & - & - \\
\hline GG & $732(81.424)$ & $560(82.474)$ & 0.128 & - & $278(79.656)$ & 0.182 & - \\
\hline TT & $11(1.224)$ & $2(0.295)$ & - & - & $1(0.287)$ & - & - \\
\hline GT & $156(17.353)$ & $117(17.231)$ & - & - & $70(20.057)$ & - & - \\
\hline G & $1620(90.100)$ & $1237(91.090)$ & 0.347 & $1.123(0.881-1.432)$ & $626(89.685)$ & 0.756 & $0.955(0.716-1.275)$ \\
\hline $\mathrm{T}$ & $178(9.900)$ & $121(8.910)$ & - & - & $72(10.315)$ & - & - \\
\hline rs1234313 & - & - & - & - & - & - & - \\
\hline AA & 365 (40.466) & 298 (43.504) & 0.474 & - & $147(41.643)$ & 0.832 & - \\
\hline GG & 110 (12.195) & 78 (11.387) & - & - & 39 (11.048) & - & - \\
\hline AG & 427 (47.339) & 309 (45.109) & - & - & $167(47.309)$ & - & - \\
\hline A & 1157 (64.135) & $905(66.058)$ & 0.261 & $1.088(0.939-1.261)$ & $461(65.297)$ & 0.584 & $1.052(0.877-1.263)$ \\
\hline G & $647(35.865)$ & 465 (33.942) & - & - & $245(34.703)$ & - & - \\
\hline rs16845607 & - & - & - & - & - & - & - \\
\hline AA & $4(0.443)$ & $4(0.581)$ & 0.897 & - & $1(0.283)$ & 0.859 & - \\
\hline GG & 797 (88.359) & $605(87.808)$ & - & - & $315(89.235)$ & - & - \\
\hline AG & $101(11.197)$ & $80(11.611)$ & - & - & $37(10.482)$ & - & - \\
\hline$A$ & $109(6.042)$ & $88(6.386)$ & 0.69 & $1.061(0.794-1.418)$ & $39(5.524)$ & 0.62 & $0.909(0.624-1.325)$ \\
\hline G & 1695 (93.958) & $1290(93.614)$ & - & - & $667(94.476)$ & - & - \\
\hline rs3850641 & - & - & - & - & - & - & - \\
\hline GG & $26(2.879)$ & $9(1.306)$ & 0.086 & - & $6(1.695)$ & 0.172 & - \\
\hline AA & $660(73.090)$ & $502(72.859)$ & - & - & $248(70.056)$ & - & - \\
\hline AG & $217(24.031)$ & 178 (25.835) & - & - & $100(28.249)$ & - & - \\
\hline G & 269 (14.895) & 196 (14.224) & 0.595 & $0.948(0.776-1.156)$ & 112 (15.819) & 0.561 & $1.074(0.845-1.364)$ \\
\hline A & 1537 (85.105) & $1182(85.776)$ & - & - & $596(84.181)$ & - & - \\
\hline
\end{tabular}

\subsection{Haplotypic Association}

Haplotypic analysis using the Haploview software (Whitehead Institute for Biomedical Research, MIT Media Lab, and Broad Institute of Harvard and MIT, Cambridge, MA, USA) revealed that in the HapMap Han Chinese Beijing database, rs7514229 and rs1234313 were in the same block (Figure 1), which contained three haplotypes, namely GA, GG and TG. However, these haplotypes were not associated with AITDs ( $p>0.05$, data not shown). 


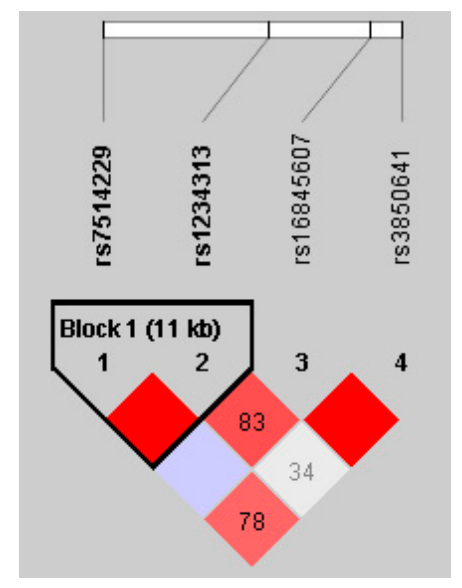

Figure 1. Linkage disequilibrium (LD) block of TNFSF4 from controls in the Hapmap CHB data.

\subsection{Genotyping-Clinical Sub-Phenotype Association}

To further investigate the relation between polymorphisms of TNFSF4 and clinical phenotypes, clinical sub-phenotype analyses were conducted. The results showed that the frequency of genotype TT in rs7514229 marginally declined in AITDs patients with disease-onset age $\geq 19$ years old ( $p=0.049$, as shown in Table 5). However, our present study displayed that TNFSF4 gene variants were not associated with AITDs patients with ophthalmopathy or family history. Interestingly, the frequency of allele G in rs3850641 was significantly more decreased in HT patients with hypothyroidism than in HT patients without hypothyroidism, suggesting that HT patients with allele G in rs3850641 had increased susceptibility risk to hypothyroidism $(p=0.018$, Table 6).

Table 5. Allele and genotype distribution of TNFSF4 in AITDs patients with or without early-onset age.

\begin{tabular}{ccccc}
\hline & \multicolumn{2}{c}{ Onset Age of AITDs Patients } & & OR (95\% CI) \\
\cline { 2 - 4 } SNP ID & $\leq \mathbf{1 8}$ & $\geq \mathbf{1 9}$ & & - \\
\hline rs7514229 & - & - & - & - \\
GG & $128(80.503)$ & $710(81.703)$ & 0.049 & - \\
TT & $2(1.258)$ & $1(0.115)$ & - & - \\
GT & $29(18.239)$ & $158(18.182)$ & - & OR \\
G & $285(89.623)$ & $1578(90.794)$ & 0.51 & - \\
T & $33(10.377)$ & $160(9.206)$ & - & - \\
rs1234313 & - & - & - & - \\
AA & $71(44.375)$ & $374(42.597)$ & 0.41 & - \\
GG & $22(13.750)$ & $95(10.820)$ & - & - \\
AG & $67(41.875)$ & $409(46.583)$ & - & - \\
A & $209(65.312)$ & $1157(65.888)$ & 0.842 & $1.026(0.799-1.318)$ \\
G & $111(34.688)$ & $599(34.112)$ & - & - \\
rs16845607 & - & - & - & - \\
AA & $1(0.625)$ & $4(0.454)$ & 0.246 & - \\
GG & $135(84.375)$ & $785(89.002)$ & - & - \\
AG & $24(15.000)$ & $93(10.544)$ & - & - \\
A & $26(8.125)$ & $101(5.726)$ & 0.099 & $0.687(0.439-1.075)$ \\
G & $294(91.875)$ & $1663(94.274)$ & - & - \\
rs3850641 & - & - & - & - \\
GG & $2(1.250)$ & $13(1.472)$ & 0.639 & - \\
AA & $120(75.000)$ & $630(71.348)$ & - & - \\
AG & $38(23.750)$ & $240(27.180)$ & - & - \\
G & $42(13.125)$ & $266(15.062)$ & 0.369 & - \\
A & $278(86.875)$ & $1500(84.938)$ & - & $-174(0.827-1.664)$ \\
\hline & & & & - \\
\hline
\end{tabular}


Table 6. TNFSF4 genotype and allele distribution in clinical sub-phenotype of HT patients.

\begin{tabular}{ccccc}
\hline \multirow{2}{*}{ TNFSF4 SNP } & \multicolumn{2}{c}{ HT } & $p$ & OR (95\% CI) \\
\cline { 2 - 4 } rs7514229 & Non-Hypothyroidism & Hypothyroidism & & - \\
GG & - & - & - & - \\
TT & $107(80.451)$ & $153(78.462)$ & 0.668 & - \\
GT & $0(0)$ & $1(0.513)$ & - & - \\
G & $26(19.549)$ & $41(21.026)$ & - & $0.874(0.523-1.462)$ \\
T & $240(90.226)$ & $347(88.974)$ & 0.608 & - \\
rs1234313 & $26(9.774)$ & $43(11.026)$ & - & - \\
AA & - & - & - & - \\
GG & $50(37.037)$ & $90(45.685)$ & 0.287 & - \\
AG & $16(11.852)$ & $19(9.645)$ & - & - \\
A & $69(51.111)$ & $88(44.670)$ & - & - \\
G & $169(62.593)$ & $268(68.020)$ & 0.148 & - \\
rs16845607 & $101(37.407)$ & $126(31.980)$ & - & - \\
AA & - & - & - & - \\
GG & $1(0.741)$ & $0.918-1.759)$ \\
AG & $115(85.185)$ & $180(91.371)$ & - & - \\
A & $19(14.074)$ & $17(8.629)$ & - & - \\
G & $21(7.778)$ & $17(4.315)$ & 0.059 & $0.535(0.277-1.034)$ \\
rs3850641 & $249(92.22)$ & $377(95.685)$ & - & - \\
GG & - & - & - & - \\
AA & $1(0.741)$ & $5(2.525)$ & 0.051 & - \\
AG & $104(77.037)$ & $129(65.152)$ & $-709(1.096-2.674)$ \\
G & $30(22.222)$ & $64(32.323)$ & - & - \\
A & $32(11.852)$ & $74(18.687)$ & 0.018 & - \\
\hline & $238(88.148)$ & $322(81.313)$ & - & - \\
\hline
\end{tabular}

\section{Discussion}

The TNFSF4 gene, also known as the OX40 ligand (OX40L), encodes the OX40L protein which is a co-stimulatory cytokine and belongs to the TNF ligand family. The protein mainly participates in the interaction of T-cell and antigen-presenting cell (APC), T-cell activation and B-cell differentiation, providing CD28-independent co-stimulatory signals for activated $\mathrm{CD}^{+} \mathrm{T}$ cells [15]. TNFSF4, located in chromosome 1 (1q25), contains three exons and two introns (in NCBI database). Previous studies have shown that polymorphisms of TNFSF4 can confer risk to diverse autoimmune diseases, such as SLE, RA, SSc and pSS, but it remains unknown whether genetic mutations of TNFSF4 region may induce occurrence of AITDs, which attracts our interest.

AITDs are also regarded as autoimmune diseases targeting the thyroid with a complex genetic and environmental etiology, manifesting mainly as GD and HT. It is notable that genetic factors play a prominent role in the occurrence and persistence of AITDs. Given that autoimmune diseases may share a common genetic predisposition, and that immune dysregulation plays a vital role in AITDs [16,17], we hypothesized that variants within the TNFSF4 gene, which is a crucial immune regulator, could also elicit abnormal OX40L expression and dysfunction, thus affecting T-cell activation and leading to unbalanced immune regulation and its resultant occurrence of AITDs.

In the present work, we observed the association between four loci of TNFSF4 gene and AITDs patients in the Han Chinese population. We found that the frequency of genotype GG in rs3850641 was slightly lower in AITDs patients, probably suggesting it could decrease susceptibility to AITDs. In addition, frequencies of GG genotype in rs3850641 and TT genotype in rs7514229 also decreased in AITDs subjects, confirming that variant genotype GG in rs3860541 was indeed a factor protecting people from AITDs, as was variant genotype TT in rs7514229. Our results suggested polymorphisms in the TNFSF4 gene region, one SNP in 3'UTR (rs7514229) and two intronic SNPs (rs3860541 and rs1234313), may be associated with AITDs susceptibility. To our knowledge, variants in the intron 
of a gene may influence its expression and regulate its function [18], 3'UTR polymorphisms in the gene region are of important regulation function. We therefore speculated that the molecular action underlying genetic pathology of AITDs is that TNFSF4 SNPs may affect the expression of TNFSF4 gene and down-regulate T-cell activation, which requires further in-depth research to confirm.

Further, to investigate the association between genotype and clinical manifestations, we carried out the clinical sub-phenotype analysis. The AITDs occurrence in teenagers ( $\leq 18$ young patients) may be due to their genetic family history of this disease $[19,20]$, which corresponded with our results showing the frequency of family history was much higher in AITDs patients with disease-onset age $\leq 18$ years old. Meanwhile, marginally significant differences in frequencies of rs7514229 genotype TT and disease-onset age were found between AITDs patients with disease-onset age $\leq 18$ years old and AITDs patients with disease-onset age $\geq 19$ years old. Similar correlations between gene mutations and disease-onset age were reported in RA [21], type 1 diabetes [22] and multiple sclerosis [23]. Furthermore, we revealed that frequency of GG genotype of rs3850641 declined slightly in GD subgroup of AITDs, although without significance. Nevertheless, we observed that TNFSF4 SNPs were not associated with AITDs patients with ophthalmopathy or family history. Several studies provided clues that thyroid-associated ophthalmopathy (TAO) was correlated with the impact of environmental elements, especially current smoking history [24,25]. Recent studies are suggesting that genetic markers also affect the susceptibility of TAO [26], including genetic variants in the STAT3 [27], TSHR [28] and HLA-DR3 [29] regions. However, our results cannot add the TNFSF4 gene to the list of the predisposition of thyroid-associated ophthalmopathy (TAO). Moreover, allele A from rs3850641 was associated with the decreased risk for the HT subgroup of hypothyroidism by $41.5 \%$. In HT, hypothyroidism is more associated with a family history of thyroid dysfunction [20]. Our study showed HT hypothyroidism patients with higher ratio of family history, which was consistent with the previous research [20]. To our best knowledge, we were the first to find that genetic factors are also involved in etiology of hypothyroidism in HT. Why do these SNPs not show their susceptibility to GD or TAO? It is possible that thyroid eye disease or TAO is a different disease than Graves' disease and Hashimoto's thyroiditis. In addition, a recent paper found that polymorphisms in calsequestrin (CASQ1) are correlated with HT and GO, but not Graves' hyperthyroiditis (GH) [30]. Interestingly, our study found SNPs in TNFSF4 are associated with hypothyroidism of Hashimoto's thyroiditis, but not thyroid orbitopathy or GD. These two studies do not show contradictory results, and illustrate the complexity of the diseases, GD, HT and TAO or GO. For instance, our previous studies indeed found UBE2L3 and CLEC16A gene polymorphisms to be associated with susceptibility to HT rather than GD and TAO or GO [2,3]. Obviously, the genetic mechanisms of these diseases are still unclear, so more research is needed to reveal the pathomechanism of thyroid ophthalmopathy.

Overall, we provided the first evidence for genetic association between four susceptibility loci in the TNFSF4 gene in Chinese AITDs patients, with samples exclusively from the Han Chinese population. Nevertheless, considering the validation of a convincing association and discovery of population differences, the importance of replication studies in some different populations should not be overlooked. The statistical power calculated in this research was very strong (larger than 0.8 ) to detect the association, and it has adequately reached a significant result. Simultaneously, the sample size in this study was large enough with 1,048 cases and 909 controls to effectively reduce the type of errors (type 1 error and type 2 error).

\section{Materials and Methods}

\subsection{Subjects}

A total of 1,048 Chinese patients with AITDs (693 GD and 355 HT) and 909 healthy Chinese controls were recruited. All AITDs patients were enrolled from the Out-Patient Department of Endocrinology of Jinshan Hospital of Fudan University. Ethnically and geographically matched and unrelated healthy controls were recruited from the Healthy Check-Up Center of the same hospital. 
All AITDs patients were diagnosed as previously described [2,27]. GD patients were diagnosed based on their clinical manifestations and biochemical assessments of hyperthyroidism and the positive circulating TRAb, with or without positive TPOAb or TgAb and diffusive goiter of the thyroid. HT was defined based on the high level of either TPOAb or TgAb, with or without clinical and biochemical hypothyroidism and the presence of an enlarged thyroid. A minority of HT patients were further confirmed by fine needle aspiration biopsies. All the control subjects showed negative thyroid antibodies against TPO. In the current study, TPOAb, TgAb and TRAb were detected with highly specific and sensitive immunochemiluminescence kits from Roche Company (Shanghai, China).

All the subjects, including AITDs patients and controls, were ethnic Han Chinese. Written informed consent was obtained from all participants and the research was approved by the Ethics Committee of Jinshan Hospital of Fudan University (JYLL-2014-06, 2014/2/21), respectively.

\subsection{DNA Sample Preparation}

Genomic DNA were extracted from $2 \mathrm{~mL}$ of peripheral venous blood from each subject using RelaxGene Blood DNA System (Tiangen Biotech Company, Beijing, China), according to the manufacturer's protocol. The concentration and A260/A280 ratio of all DNA samples were measured by NANO DROP 2000 Spectrophotometer (Thermo Scientific Company, Waltham, MA, USA). Finally, the DNA samples with great purity and concentration were used for next genotyping.

\subsection{Single Nucleotide Polymorphism (SNP) Selection and Genotyping}

Marker-tagging SNPs were chosen from the Hapmap CHB data using the Tagger programme of Haploview software (Whitehead Institute for Biomedical Research, MIT Media Lab, and Broad Institute of Harvard and MIT) to satisfy the following criteria: minor allele frequency (MAF) $>0.1$, Hardy-Weinberg equilibrium (HWE) with $p>0.001$ and logarithm of odds (LOD) $>3.0$. For the TNFSF4 gene of $23 \mathrm{~kb}$ with 42 SNPs in Hapmap CHB population, we selected four loci covering the whole region of the TNFSF4 gene to capture all the most common variants. Four tag SNPs were selected including rs7514229 located in the $3^{\prime}$ untranslated region (UTR), as well as rs1234313, rs16845607 and rs3850641 in intron 1 of the TNFSF4 region.

Genotyping of the four SNPs was undertaken using the ligase detection reaction (LDR) platform according to the manufacturer's instructions. Moreover, to ensure detection quality, each reaction was performed in duplicate, and blank samples without DNA were used as negative controls. Furthermore, only SNPs and samples that passed the $95 \%$ quality control threshold were subjected to further statistical analysis and SNPs with allele frequencies not meeting Hardy-Weinberg equilibrium (HWE) were removed from the next analysis. The primers specific to the four SNPs at the TNFSF4 loci are "rs7514229" forward-GATAACACAGAATCATCCAG and reverse-TTGTAGCACATGTTTCCCTG; "rs1234313" forward-ATCTAACACTGGCTCTAGTC and reverse-GCCATTCTGACTAGAATAGG; "rs16845607" forward-AGATATAGCTACCAAGCTCC and reverse-GATGAGAAAACAGAGGCTAC; "rs3850641" forward-GCTGTCACTTTGAAGCTTTG and reverse-TGCCTGATCAAACACATTAC.

\subsection{Clinical Sub-Phenotype Analysis}

Clinical sub-phenotype stratification analysis was conducted using a case-only approach, in which basic allelic and genotypic examination was performed by comparing minor allele and genotype frequency of cases with a specific sub-phenotype to the whole case group. The clinical sub-phenotypes include: (1) the age of disease onset ( $\leq 18$ years old versus $\geq 19$ years old); (2) presence or absence of ophthalmopathy which was defined as a distinctive disorder characterized by inflammation and swelling of the extraocular muscles, eyelid retraction, periorbital edema, episcleral vascular injection, conjunctive swelling and proptosis; (3) presence or absence of hypothyroidism in HT patients; and (4) presence or absence of AITDs family history, which was defined as the subjects' first-degree relatives including parents, children and siblings or second-degree relatives such as grandparents, uncles and aunts who had AITDs. 


\subsection{Statistical Analysis}

Clinical data were described as $M \pm S D$ (mean \pm standard deviation). Hardy-Weinberg equilibrium (HWE) concordance test in the controls and patient samples, linkage disequilibrium (LD) test and haplotype frequency calculation were performed using HaploView 4.2 (Whitehead Institute for Biomedical Research, MIT Media Lab, and Broad Institute of Harvard and MIT). In order to analyze whether the four predisposing loci are associated with AITDs, allele and genotype frequencies were compared between AITDs cases and healthy controls using the Chi-square test $\left(\chi^{2}\right.$-test) or Fisher's exact test. LD among the selected SNPs was measured using the pairwise LD measures $D^{\prime}$ and $r^{2}$. All data were statistically calculated with the SPSS 18.0 software (International Business Machines Corporation, Armonk, NY, USA). A $p$ value of less than 0.05 was considered statistically significant. Odds ratio (OR) and 95\% confidence interval $(95 \% \mathrm{CI})$ were applied to assess the association between each genotype and AITDs.

\subsection{Power Calculation}

Power calculations for AITDs in this research considered allele frequency of SNPs from 0.05 to 0.5 , a population prevalence of $1 \%-5 \%$ for AITDs, and OR of $0.2-0.5$ at a 0.05 significant level. As a result, this study had sufficient power (larger than 0.8 ) to detect the association of $O R$ of 0.2 or above with 1,048 cases and 909 controls.

\section{Conclusions}

In conclusion, the preliminary findings of our present study are the first to indicate the association of novel genetic susceptibility loci of the TNFSF4 region with the predisposition to AITDs. Additionally, our results support the importance of T cells in the pathology of AITDs, and reveal the frequency overlap of risk loci in immune pathways between AITDs and other autoimmune diseases.

Acknowledgments: This project is supported by grants from the National Natural Science Foundation of China (No. 81471004) and the Key Disciplines Development of Shanghai Jinshan District (No. JSZK2015A02). The authors would like to thank all of the people who took part in the study.

Author Contributions: Rong-Hua Song and Qiong Wang carried out the work and contributed equally to the work. Qiu-Ming Yao, Xiao-Qing Shao, Ling Li, Wen Wang, Xiao-Fei An and Qian Li helped specimens collection. Rong-Hua Song conducted the data analysis and wrote the manuscript. Jin-An Zhang designed the study and coordinated the research team.

Conflicts of Interest: The authors declare no conflict of interest.

\section{References}

1. Tomer, Y. Mechanisms of autoimmune thyroid diseases: From genetics to epigenetics. Annu. Rev. Pathol. 2014, 9, 147-156. [CrossRef] [PubMed]

2. Muhali, F.S.; Cai, T.T.; Zhu, J.L.; Qin, Q.; Xu, J.; He, S.T.; Shi, X.H.; Jiang, W.J.; Xiao, L.; Li, D.F.; et al. Polymorphisms of CLEC16A region and autoimmune thyroid diseases. G3 (Bethesda) 2014, 4, 973-977. [CrossRef] [PubMed]

3. Wang, Y.; Zhu, Y.F.; Wang, Q.; Xu, J.; Yan, N.; Xu, J.; Shi, L.F.; He, S.T.; Zhang, J.A. The haplotype of UBE2L3 gene is associated with Hashimoto's thyroiditis in a Chinese Han population. BMC Endocr. Disord. 2016, 16, 18-23. [CrossRef] [PubMed]

4. Balazs, C. The role of hereditary and environmental factors in autoimmune thyroid diseases. Orv. Hetil. 2012, 153, 1013-1022. [CrossRef] [PubMed]

5. Effraimidis, G.; Wiersinga, W.M. Mechanisms in endocrinology: Autoimmune thyroid disease: Old and new players. Eur. J. Endocrinol. 2014, 170, 241-252. [CrossRef] [PubMed]

6. Orozco, G.; Eyre, S.; Hinks, A.; Bowes, J.; Morgan, A.W.; Wilson, A.G.; Wordsworth, P.; Steer, S.; Hocking, L.; Thomson, W.; et al. Study of the common genetic background for rheumatoid arthritis and systemic lupus erythematosus. Ann. Rheum. Dis. 2011, 70, 463-468. [CrossRef] [PubMed] 
7. Gourh, P.; Arnett, F.C.; Tan, F.K.; Assassi, S.; Divecha, D.; Paz, G.; McNearney, T.; Draeger, H.; Reveille, J.D.; Mayes, M.D.; et al. Association of TNFSF4 (OX40L) polymorphisms with susceptibility to systemic sclerosis. Ann. Rheum. Dis. 2010, 69, 550-555. [CrossRef] [PubMed]

8. Graham, R.R.; Cotsapas, C.; Davies, L.; Hackett, R.; Lessard, C.J.; Leon, J.M.; Burtt, N.P.; Guiducci, C.; Parkin, M.; Gates, C.; et al. Genetic variants near TNFAIP3 on 6q23 are associated with systemic lupus erythematosus. Nat. Genet. 2008, 40, 1059-1061. [CrossRef] [PubMed]

9. Bowes, J.; Lawrence, R.; Eyre, S.; Panoutsopoulou, K.; Orozco, G.; Elliott, K.S.; Ke, X.; Morris, A.P.; Thomson, W.; Worthington, J.; et al. Rare variation at the TNFAIP3 locus and susceptibility to rheumatoid arthritis. Hum. Genet. 2010, 128, 627-633. [CrossRef] [PubMed]

10. Dieude, P.; Guedj, M.; Wipff, J.; Ruiz, B.; Riemekasten, G.; Matucci-Cerinic, M.; Melchers, I.; Hachulla, E.; Airo, P.; Diot, E.; et al. Association of the TNFAIP3 rs5029939 variant with systemic sclerosis in the european caucasian population. Ann. Rheum. Dis. 2010, 69, 1958-1964. [CrossRef] [PubMed]

11. Song, R.H.; Yu, Z.Y.; Wang, Q.; Muhali, F.S.; Jiang, W.J.; Xiao, L.; Shi, X.H.; He, S.T.; Xu, J.; Zhang, J.A. Polymorphisms of the TNFAIP3 region and Graves' disease. Autoimmunity 2014, 47, 459-465. [CrossRef] [PubMed]

12. Zhou, X.J.; Lu, X.L.; Nath, S.K.; Lv, J.C.; Zhu, S.N.; Yang, H.Z.; Qin, L.X.; Zhao, M.H.; Su, Y.; Shen, N.; et al. Gene-gene interaction of BLK, TNFSF4, TRAF1, TNFAIP3, and REL in systemic lupus erythematosus. Arthritis Rheum. 2012, 64, 222-231. [CrossRef] [PubMed]

13. Coustet, B.; Bouaziz, M.; Dieude, P.; Guedj, M.; Bossini-Castillo, L.; Agarwal, S.; Radstake, T.; Martin, J.; Gourh, P.; Elhai, M.; et al. Independent replication and meta analysis of association studies establish TNFSF4 as a susceptibility gene preferentially associated with the subset of anticentromere-positive patients with systemic sclerosis. J. Rheumatol. 2012, 39, 997-1003. [CrossRef] [PubMed]

14. Sun, F.; Li, P.; Chen, H.; Wu, Z.; Xu, J.; Shen, M.; Leng, X.; Shi, Q.; Zhang, W.; Tian, X.; et al. Association studies of TNFSF4, TNFAIP3 and FAM167A-BLK polymorphisms with primary Sjogren's syndrome in Han Chinese. J. Hum. Genet. 2013, 58, 475-479. [CrossRef] [PubMed]

15. Kinnear, G.; Wood, K.J.; Fallah-Arani, F.; Jones, N.D. A diametric role for OX40 in the response of effector/memory CD4 ${ }^{+} \mathrm{T}$ cells and regulatory $\mathrm{T}$ cells to alloantigen. J. Immunol. 2013, 191, 1465-1475. [CrossRef] [PubMed]

16. Simmonds, M.J. GWAS in autoimmune thyroid disease: Redefining our understanding of pathogenesis. Nat. Rev. Endocrinol. 2013, 9, 277-287. [CrossRef] [PubMed]

17. Marique, L.; Van Regemorter, V.; Gerard, A.C.; Craps, J.; Senou, M.; Marbaix, E.; Rahier, J.; Daumerie, C.; Mourad, M.; Lengele, B.; et al. The expression of dual oxidase, thyroid peroxidase, and caveolin-1 differs according to the type of immune response (Th1/Th2) involved in thyroid autoimmune disorders. J. Clin. Endocrinol. Metab. 2014, 99, 1722-1732. [CrossRef] [PubMed]

18. Alberobello, A.T.; Congedo, V.; Liu, H.; Cochran, C.; Skarulis, M.C.; Forrest, D.; Celi, F.S. An intronic SNP in the thyroid hormone receptor $\beta$ gene is associated with pituitary cell-specific over-expression of a mutant thyroid hormone receptor $\beta 2(\mathrm{R} 338 \mathrm{~W})$ in the index case of pituitary-selective resistance to thyroid hormone. J. Transl. Med. 2011, 9, 144-151. [CrossRef] [PubMed]

19. Brix, T.H.; Petersen, H.C.; Iachine, I.; Hegedus, L. Preliminary evidence of genetic anticipation in Graves' disease. Thyroid 2003, 13, 447-451. [CrossRef] [PubMed]

20. Manji, N.; Carr-Smith, J.D.; Boelaert, K.; Allahabadia, A.; Armitage, M.; Chatterjee, V.K.; Lazarus, J.H.; Pearce, S.H.; Vaidya, B.; Gough, S.C.; et al. Influences of age, gender, smoking, and family history on autoimmune thyroid disease phenotype. J. Clin. Endocrinol. Metab. 2006, 91, 4873-4880. [CrossRef] [PubMed]

21. Radstake, T.R.; Barrera, P.; Albers, M.J.; Swinkels, H.L.; van de Putte, L.B.; van Riel, P.L. Genetic anticipation in rheumatoid arthritis in Europe. European consortium on rheumatoid arthritis families. J. Rheumatol. 2001, 28, 962-967. [PubMed]

22. Paterson, A.D.; Kennedy, J.L.; Petronis, A. Evidence for genetic anticipation in non-mendelian diseases. Am. J. Hum. Genet. 1996, 59, 264-268. [PubMed]

23. Cocco, E.; Sardu, C.; Lai, M.; Spinicci, G.; Contu, P.; Marrosu, M.G. Anticipation of age at onset in multiple sclerosis: A sardinian cohort study. Neurology 2004, 62, 1794-1798. [CrossRef] [PubMed] 
24. Villanueva, R.; Inzerillo, A.M.; Tomer, Y.; Barbesino, G.; Meltzer, M.; Concepcion, E.S.; Greenberg, D.A.; MacLaren, N.; Sun, Z.S.; Zhang, D.M.; et al. Limited genetic susceptibility to severe Graves' ophthalmopathy: No role for CTLA-4 but evidence for an environmental etiology. Thyroid 2000, 10, 791-798. [CrossRef] [PubMed]

25. Melcescu, E.; Horton, W.B.; Kim, D.; Vijayakumar, V.; Corbett, J.J.; Crowder, K.W.; Pitman, K.T.; Uwaifo, G.I.; Koch, C.A. Graves orbitopathy: Update on diagnosis and therapy. South. Med. J. 2014, 107, 34-43. [CrossRef] [PubMed]

26. Li, H.; Chen, Q. Genetic susceptibility to Grave's disease. Front. Biosci. 2013, 18, 1080-1087. [CrossRef]

27. Xiao, L.; Muhali, F.S.; Cai, T.T.; Song, R.H.; Hu, R.; Shi, X.H.; Jiang, W.J.; Li, D.F.; He, S.T.; Xu, J.; et al. Association of single-nucleotide polymorphisms in the STAT3 gene with autoimmune thyroid disease in chinese individuals. Funct. Integr. Genom. 2013, 13, 455-461. [CrossRef] [PubMed]

28. Liu, L.; Wu, H.Q.; Wang, Q.; Zhu, Y.F.; Zhang, W.; Guan, L.J.; Zhang, J.A. Association between thyroid stimulating hormone receptor gene intron polymorphisms and autoimmune thyroid disease in a Chinese Han population. Endocr. J. 2012, 59, 717-723. [CrossRef] [PubMed]

29. Jurecka-Lubieniecka, B.; Ploski, R.; Kula, D.; Krol, A.; Bednarczuk, T.; Kolosza, Z.; Tukiendorf, A.; Szpak-Ulczok, S.; Stanjek-Cichoracka, A.; Polanska, J.; et al. Association between age at diagnosis of Graves' disease and variants in genes involved in immune response. PLoS ONE 2013, 8, e59349.

30. Lahooti, H.; Cultrone, D.; Edirimanne, S.; Walsh, J.P.; Delbridge, L.; Cregan, P.; Champion, B.; Wall, J.R. Novel single-nucleotide polymorphisms in the calsequestrin-1 gene are associated with Graves' ophthalmopathy and Hashimoto's thyroiditis. Clin. Ophthalmol. 2015, 9, 1731-1740. [CrossRef] [PubMed]

(C) 2016 by the authors; licensee MDPI, Basel, Switzerland. This article is an open access article distributed under the terms and conditions of the Creative Commons Attribution (CC-BY) license (http:/ / creativecommons.org/licenses/by/4.0/). 